\title{
EL CARÁCTER ANDALUZ DURANTE CUATRO DÉCADAS DE DEMOCRACIA: IDENTIDADES TERRITORIALES Y CULTURAS POLÍTICAS 1
}

\author{
Francisco Collado Campaña \\ Universidad Pablo de Olavide, Sevilla
}

http://dx.doi.org/10.5209/NOMA.51641

\begin{abstract}
Resumen: En 2015 se han cumplido cuarenta años de democracia representativa en España. A lo largo de este período, el desarrollo del Estado de las autonomías ha configurado un heterogéneo poliedro con distintas comunidades, identidades y formas de concebir la vida política en cada territorio. En el caso andaluz, han existido dos dinámicas en torno al imaginario de cómo son vistos y cómo se ven a sí mismos sus habitantes. Primero, una imagen negativa del andaluz promovida por algunos sectores de la opinión pública frente a otra positiva que destaca sus avances económicos, sociales y culturales. Segundo, un conflicto interno (a veces silencioso) entre las distintas identidades territoriales existentes en el interior de la comunidad. Ante esta variedad de opiniones, se presenta un análisis diacrónico de las identidades territoriales (europea, nacional, autonómica y local) y la cultura política andaluces a partir de los principales datos estadísticos del Centro de Estudios Andaluces, CIS e IESA.
\end{abstract}

Palabras clave: Andalucía, identidad, situación política, ideología, materialismo, postmaterialismo.

Abstract: In 2015, ago forty years of representative democracy in Spain. Throughout this period, the development of the State of the autonomies has formed a heterogeneous polyhedron with different communities, identities and ways of conceiving the political life in every territory. In the andalusian case, two dynamics have existed concerning the imaginary one of how they are seen and how the same his inhabitants see themselves. First, a negative image of the andalusian constructed by some actors of the public opinion opposite to positive other one that emphasizes his economic, social and cultural advances. Second, an internal (and often silent) conflict between the different territories in the set of eight provinces. Before this variety of opinions, we presents a diachronical analysis of the territorial identities of the andalusian inhabitants and the political culture from the principal statistical dates of Center of Andalusian Studies, CIS and IESA.

Keywords: Andalusia, identity, political context, ideology, materialism, postmaterialism.

\section{Introducción}

¿Qué significa ser andaluz? ¿Cómo es la ciudadanía andaluza? Para algunos el imaginario sobre este territorio sigue vistiéndose con elementos folclóricos y étnicos asociados al flamenco, la tauromaquia,

1 El presente artículo es el desarrollo de un paper previo en el que se expone una visión diacrónica de la identidad política y la cultura política de Andalucía desde 1975 hasta 2015 a partir de distintas obras, bases de datos y reflexiones sobre dicho tema de investigación. 
la inmigración y el mundo rural ${ }^{2}$. Para otros bien conocedores de su heterogénea realidad, es una tarea ardua definir un mínimo común que permita agrupar desde el habitante de las áreas urbanas de Sevilla, Málaga y Cádiz hasta el andaluz de las áreas más rurales y despobladas situadas en lugares como la Sierra de Aracena y la Alpujarra granadina. En este sentido, surge la pregunta de cuál es la identidad territorial o identidades territoriales que definen a los andaluces en los tiempos más recientes.

En los últimos años, se han venido desarrollando dos opiniones sobre la población andaluza. En un primer lugar, una opinión negativa que ha venido a destacar aquellos elementos que suelen asociarse con los andaluces como poco trabajadores, juerguistas, hipócritas, incultos o que hacen un uso incorrecto ${ }^{3}$ de la lengua española, entre otros. Al respecto, se pueden mencionar las críticas que en distintas ocasiones han recibido de distintos sectores de la clase política, por ejemplo, unas declaraciones de Duran i Lleida sostenía que "los andaluces se pasan el día en el bar" en 2011 cuando se manifestó en referencia a los agricultores catalanes ${ }^{4}$. Tras esta polémica, se detectó que los adjetivos "vagos", "analfabetos" y "falsos" eran los calificativos que aparecían con más frecuencia en los buscadores de Internet. Así, una empresa andaluza de publicidad Ilamada "Pura Envidia" lanzó una campaña para cambiar este panorama 5 . La metodología que se seleccionó fue el lanzamiento de búsquedas positivas desde Google para modificar los indexadores.

En segundo lugar, existen otros calificativos positivos vinculados al estereotipo andaluz. Entre ellos, se encuentran su imagen asociada a las festividades como la Semana Santa y las ferias populares, pero que no ayudan a romper con la imagen habitual de esta comunidad. Al respecto, algunas investigaciones han destacado como gran parte de la ciudadanía andaluza tiene predilección por la participación asociativa en colectivos de carácter musical, religioso y/o deportivo

2 En una entrevista realizada al profesor Félix Moral de la Facultad de Psicología de la Universidad de Málaga se exponía cómo estos estereotipos afectaban especialmente al aumento de la visibilidad andaluza frente a otras realidades territoriales en España. Más información: http://www.laopiniondemalaga.es/malaga/2010/1 1/21/andalucia-prisioneraestereotipos/382834.html

3 Esta crítica se fundamenta en el desacuerdo existente entre los lingüistas sobre la existencia de un dialecto andaluz. Entre aquellos que afirman la existencia de dicho dialecto existen controversias sobre la clasificación de sus variedades geográficas. Por eso, lo que algunos consideran un dialecto, para otros es un uso que rompe con la norma de la lengua.

4 Para más información, se puede consultar esta información: http://politica.elpais.com/politica/201 1/10/08/actualidad/1318090413_762077.html

5 Se puede consultar más datos sobre dicha campaña en la página web que se habilitó para dicho fin: http://www.losandalucesson.com/. En el momento en el que se redactaba el presente artículo se han contabilizado 235.921 búsquedas con valoraciones positivas sobre la población andaluza. 
(Moyano y Pérez-Yruela, 2002). Sin embargo, estas dinámicas tienden a crear una imagen muy generalista de una comunidad autónoma de 8.402.305 de habitantes para fechas de 2014, según datos del INE.

En el año 2015, se cumplen cuatro décadas del inicio de la Transición que no sólo trajo consigo la democracia, sino también el desarrollo del Estado de las autonomías (Linz, 1985). En este sentido, Andalucía fue una de las regiones pioneras en solicitar su reconocimiento como comunidad autónoma a finales de los setenta tras las reivindicaciones nacionalistas de Cataluña y País Vasco. El 4 de diciembre de 1977, la clase política y la socie dad civil organizada en la llamada Asamblea de Parlamentarios convocó distintas manifestaciones para la creación de la autonomía (Cuenca, 1984; De los Santos, 2002). Como compromiso de la clase política andaluza, se redactaron los llamados "Pactos de Antequera" que recogían: un proyecto de junta pre-autonómica, proponían a la localidad homónima como capital y se suscribía el apoyo de distintas fuerzas políticas. Finalmente, la pre-autonomía fue aceptada un año más tarde, siendo nombrado Plácido Fernández Viagas como primer Presidente de la misma, iniciando un proceso de constitución y reconociendo Sevilla como capital administrativa. A principios de la década de los ochenta, tras el referéndum celebrado se reconocía a Andalucía como una comunidad autónoma (Ruiz Romero, 2002).

Es entonces, cuando cabe preguntarse: ¿̇cómo han cambiado los valores de los andaluces y los sentimientos de pertenencia a esta comunidad? Especialmente, cuando existe una generación que ha nacido en la democracia que convive junto con otra que conoció los anteriores episodios históricos. A la par, existen discrepancias identitarias dentro de los andaluces de distintas provincias y localidades. ¿Cómo se ven a sí mismos y cómo se ve un andaluz de un lugar frente a otro de otra provincia? Esta otra pregunta tampoco debe ser desechada en el momento presente, ya que aunque de forma testimonial o anecdótica han surgido determinados movimientos que defienden la fragmentación territorial de la comunidad autónoma. Al respecto, cabe preguntarse cuál es la visión que tienen de la situación política andaluza, cómo es ideológicamente el ciudadanos andáluz o cuáles son los valores imperantes en su fuero interno.

\section{Objetivos}

Después de cuatro décadas de democracia y la consolidación de Andalucía como una comunidad dentro del Estado de las autonomías, estos debates siguen vigentes. ¿̇Cómo es el ciudadano andaluz en

6 Entre estos colectivos se pueden mencionar la Plataforma por Andalucía Oriental (Almería, Granada y Jaén) y la Asociación Rayya en Málaga que defiende la creación de una comunidad uniprovincial. 
cuanto a las identidades territoriales que le definen?, ¿̇y cuáles son las actitudes imperantes hacia la esfera política más allá de consignas partidistas? Éstas son las dos preguntas de investigación principales del presente artículo y que se desglosan en las siguientes preguntas secundarias:

- Identidades territoriales:

¿Cuál es el grado de los sentimientos de pertenencia de los andaluces con respecto a España y la Comunidad Autónoma de Andalucía?

- $\quad$ ¿Cuáles son los sentimientos de pertenencia de la población andaluza con respecto a los niveles de gobierno provincial y municipal7?

- ¿ ¿Cuál es el grado de sentimiento de pertenencia de los andaluces con respecto a la Unión Europea?

- Cultura política:

¿Cómo los andaluces valoran la realidad política de su comunidad autónoma?

- $\quad$ ¿Cuál es el grado de posicionamiento de los andaluces en el eje ideológico izquierda-derecha?

- ¿Cuáles son los grados de valores postmaterialistas y materialistas entre la pbolación andaluza?

De esta forma, la presente investigación pretende arrojar una luz sobre estas dudas que permitan ofrecer una imagen más analítica y alejada del imaginario en torno a los andaluces. Con ello, se podrá conocer cómo las transformaciones políticas, económicas y sociales prodúcidas por la democracia han venido a definir nuevas identidades y a consolidar una cultura en torno a la realidad política y social. Al respecto, ante la visión generalista de los estereotipos (tanto positivos como negativos) se aspira a una mayor concreción que recoja la diversidad de caracteres y actitudes de una de las comunidades con mayor peso demográfico y extensión geográfica en el conjunto del Estado español.

7 En este caso, no es posible medir el grado de pertenencia de los andaluces con respecto a su provincia o localidad debido a la ausencia de estos datos desagregados por parte de los principales institutos demoscópicos y estadísticos. Además, en el presente artículo se efectúa un posicionamiento en defensa de estudios y análisis que permitieran conocer estos datos. 


\section{Metodología}

En relación a los anteriores interrogantes de investigación, se propone realizar una visión diacrónica de las identidades territoriales y la cultura política de los andaluces desde 1975 hasta 2012. Para ello, se procede principalmente a un análisis de series temporales y diversos barómetros que se han realizado hasta el momento sobre estos objetos de investigación. En aquellos casos en los que no están disponibles las series temporales se ha recurrido a un análisis sincrónico a través de datos procedentes de una fecha determinada. La mayor parte de estos análisis proceden de instituciones como el Centro de Investigaciones Sociológicas (CIS), el Instituto de Estudios Sociales Avanzados (IESA) y del Centro de Estudios Andaluces. Sin embargo, se debe advertir que debido a la dispersión de series y datos cuantitativos se presentan los mismos desde el año en que se recogieron por primera vez. Por lo que, las series no se inician necesariamente en el año de inicio del período que se pretende abarcar.

Estos datos estadísticos se presentan desglosados a nivel andaluz en la medida de lo posible. No obstante, algunos datos puntuales no han podido ser analizados a nivel autonómico debido a que dicho desglose no está disponible por parte de las anteriores instituciones de investigación. La estructura de los resultados del artículo se desarrolla de la siguiente forma: un primer apartado dedicado a los sentimientos de pertenencia territoriales (nacional, andaluz, local y europeo); un segundo dedicado a las actitudes políticas sobre las preocupaciones de los andaluces con respecto a su comunidad, la ideología y el debate materialismo-postmaterialismo; y finalmente, se realiza una valoración global de dichos datos para el conjunto del período temporal abarcado.

De esta forma, se pretenden asentar algunas luces sobre las anteriores cuestiones en una visión crítica de la modernidad. Según Francisco Entrena (2001: 261-267), los procesos de modernización no se producen de forma simultánea ni siguen unos procesos marcados por una regularidad. Por eso, cualquier análisis que se precie de estudiar la modernidad en un territorio debe tener en cuenta tres dimensiones: socio-económica, destinada a la organización de los factores productivos y la estructura social; político-económica, correspondiente con la normalización de la violación legítima; y la simbólico-cultural, que explica y/o justifica el status quo existente. En esta lógica, este artículo represente un análisis de la dimensión "simbólico-cultural" de la modernidad en el territorio andaluz. 


\section{Identidad andaluza, identidades locales y ciudadanía europea: de lo étnico a lo territorial y de lo territorial a lo político}

En este apartado, se aborda la controversia sobre la identidad andaluza. Para ello, se presenta una situación del estado actual del andalucismo, un recorrido histórico sobre las diversas culturas que han poblado Andalucía, el sentimiento andaluz y su relación con el español, las identidades locales y el sentimiento de pertenencia a la Unión Europea. De esta forma, se puede visualizar claramente la situación de la identidad andaluza y otros sentimientos de pertenencia existentes entre los andaluces.

Se define la identidad como un elemento con una variante étnicolingüística o cultural, formada por la existencia de una lengua o dialecto propio y/o una historia, un pasado, un folclore y una cultura que define a un grupo frente otros; y otra variante cívica, en la que el grupo se caracteriza por pertenecer a una comunidad con unas normas y unos valores comunes (Linz, 1985). Ambas dimensiones pueden ser excluyentes o complementarias según cada caso (territorio, nación, etc.).

\subsection{Idendidad andaluza y española: entre etnicismo y civismo}

El concepto de identidad andaluza representa un elemento de difícil definición y muy a menudo, es objeto de controversia si se puede hablar de regionalismo o de nacionalismo andaluz. Al respecto, la obra de Blas Infante (2008) desarrollada a principios del siglo XX se considera el principal corpus teórico en el que se recogen las ideas y teorías sobre la "nación andaluza"8. Más tarde, otros como Alejandro Rojas Marcos, Diego de los Santos y Miguel Ángel Arredonda, por citar algunos, han contribuido intelectualmente a la producción de obras que profundizan en la identidad andaluza y que se consideran a sí mismos continuadores de la tradición andalucista de Infante (Rojas Marcos, 2004: 178). Andalucía a diferencia de otros territorios como Cataluña, País Vasco y Galicia no ha llegado a configurar un discurso común sobre qué significa ser andaluz o cuáles son las señas de identidad de Andalucía, según sostienen algunos autores (Aguiar y Espinosa, 2011; García Faroldi, 2008; Del Pino y Bericat, 1996). Entre los factores que han limitado la consolidación de un marco o "framing" homogéneo de lo andaluz se encuentran los siguientes:

8 La obra de Infante ha sido sacralizada por destacar el papel de Andalucía como nación. Si bien, existen determinados aspectos intelectuales y biográficos que son objeto de controversia en este autor, por ejemplo, cuando habla de las raíces musulmanas de Al-Andalus, a lo que autores como Carlos Castilla se han opuesto defendiendo la "castellanización" del territorio. 
- La existencia de sentimientos de orgullo o identidades como "ser español" o "ser de mi ciudad" que se anteponen o se mantienen jerárquicamente en la misma posición que "ser andaluz", según distintas encuestas y estudios (Aguiar y Espinosa, 2011; Coller, 2014).

- $\quad$ El desarrollo de un imaginario "sobre lo andaluz" creado por la visión decimonónica9 de los aventureros británicos (Washington Irving, William Jacob, Elizabeth Lady Holland, etc.) sobre la mistificación de la historia andaluza (Moreno Navarro, 1994) y por las políticas culturales y audiovisuales de la dictadura, que crearon un imaginario de lo español a partir de lo andaluz (Hijano del Río y Martín Zuñiga, 2007; Ruiz Muñoz y Sánchez Alarcón, 2008; Sánchez Alarcón, Díaz, Martín y Ruiz, 2007).

- El fallido intento de articulación de un partido político de corte regionalista y/o nacionalista en torno a lo andaluz, como se observa en el Partido Andalucista (PA) y en el Partido Socialista de Andalucía (PSA), en las últimas décadas (Jerez, 1985; Pérez Nieto, 2006). Por otro lado, el partido político de Rojas Marcos no fue tanto una continuidad de la postura de Blas Infante, sino que incorporó dichos planteamientos teóricos y las juntas liberalistas andaluzas años más tarde de comenzar su oposición organizada al régimen franquista (Rojas Marcos, 2004: 154).

- En relación con el anterior punto, la ausencia de una continuidad intelectual y teórica sobre el andalucismo de Blas Infante después de la Guerra Civil. A diferencia de casos como Cataluña, donde la Renaixença y su estudio junto con la defensa del catalán continuaron de la mano de Joan Triadú, Ramón Gali o Albert Manent, entre otros, durante la dictadura (Rovira, 1990; Vega, 2010). De hecho, un estudio de García Faroldi (2009: 932-933) demuestra cómo la historia política más reciente de Cataluña y Andalucía han llevado a reafirmar estas posiciones. En el caso catalán, el tripartito existente en la Generalidad a principios de la década pasada produjo un aumento del sentimiento catalanista y una nueva ola de demandas por parte de Cataluña hacia el Gobierno Central. Mientras que, en cambio Andalucía mantuvo su primacía de sentimientos igualados tanto hacia el territorio autonómico como hacia el español.

Por estos motivos, es complicado afirmar que exista una identidad andaluza, cuanto menos es innegable que dicha identidad no se ha consolidado. Y en esta disyuntiva, surge la pregunta en torno a qué es Andalucía más allá de la demarcación territorial de dicha comunidad autónoma. Si bien, existe un acuerdo teórico sobre la aportación cultural de los pueblos y las civilizaciones de este territorio y su importancia en la Historia, teniendo en cuenta que ha sido uno de los

9 Se puede consultar un estudio sobre las obras de distintos intelectuales británicos sobre sus viajes por la Andalucía del siglo XIX en la siguiente página: http://www.bibliotecavirtualdeandalucia.es/opencms/viaje-memoria/viaje002.html 
principales puntos geográficos de desarrollo político, económico y social desde sus orígenes mediterráneos en la Edad Antigua ${ }^{10}$.

Según la postura sostenida por Isidoro Moreno, Andalucía ha destacado por el mantenimiento de una civilización propia y autóctona que ha incorporado distintos elementos de los pueblos que la han habitado y a la que define como "la civilización más antigua de Europa" (Moreno Navarro, 1998: 1-2). En esa lógica, el reino de Tartessos es la primera cultura de la que se tiene constancia en tierras andaluzas y la presencia de los pueblos íberos, celtas y celtíberos. Esta civilización se desarrolló a principios del primer milenio antes de Cristo en el Bajo Guadalquivir. Además, durante ese período los fenicios fundaron distintas factorías como Gadir (Cádiz), Malaka (Málaga), Sexi (Almuñecar) y Abdera (Adra). Más tarde, la llegada de los cartagineses, pueblo de influencia fenicia/semítica y su derrota en las Guerras Púnicas (s. III a.C.) favorecieron la rápida romanización del territorio, constituyendo la provincia Bética (Moreno Navarro, 1994).

Con la consiguiente caída del Imperio Romano en el siglo IV, la región andaluza quedó bajo el control de los reinos visigóticos, a quienes hipotéticamente se debe el origen del nombre de la región ${ }^{11}$. Si bien, durante un breve período de tiempo, Bizancio ocupó una franja costera desde Huelva hasta Cartagena, formando la provincia de Spania y en un intento por recuperar el antiguo legado romano (Collado-Campaña, 2012). Durante la Edad Media (s. VIII - XV), los musulmanes conquistaron la mayor parte de la Península lbérica y crean Al-Andalus con Córdoba como capital política y se mantiene dicha presencia hasta la caída del Reino Nazarí de Granada. En el Renacimiento, Andalucía se divide en la Alta y Baja Andalucía dentro de España como Estado moderno y manteniéndose de forma ininterrumpida hasta la actualidad (Moreno Navarro 1998). Andalucía juega un papel importante en la colonización española de América debido a los puertos de Sevilla y Cádiz, donde se centraliza el comercio con las Indias. Una última aportación cultural vendrá de mano de la presencia inglesa en las costas malagueñas y gaditanas, introduciendo las ideas liberales y el protestantismo durante el siglo XIX.

Pese al legado histórico expuesto, el sentimiento de pertenencia equilibrado tanto a Andalucía como a España es la opinión mayoritaria entre los andaluces a lo largo de los años de democracia. Así, se puede

10 De hecho, en no pocas ocasiones, los representantes políticos y los intelectuales de la democracia argumentan y han argumentado la singularidad social y cultural andaluza o local aludiendo a estos episodios históricos. Buena parte de la legitimidad de los alcaldes de los municipios andaluces descansa sobre la referencia a reminiscencias del pasado, la identidad de la ciudad y/o la idiosincracia de su historia. 11 Existen multitud de teorías sobre el nombre de "Al-Andalus" o "Andalucía". Según una hipótesis podría estar en la palabra "Vandalicia" o "tierra de vándalos", una segunda tesis sostiene que puede estar en la palabra visigótica "Landa-hlauts" y una tercera sostiene que proviene de la palabra griega "Atlantis". 
observar en la siguiente serie del CIS sobre sentimiento nacionalista en Andalucía (Gráfico 1). Cabe reseñar que esta opinión no ha sido inferior al $50 \%$ en las más de tres décadas de existencia de la comunidad autónoma. Mientras que las posiciones situadas en alguno de los extremos, los que se consideran más andaluces que españoles, más españoles que andaluces, sólo andaluces y sólo españoles se han mantenido constantes por debajo del $15 \%$, ni siquiera durante principios de los ochenta, momento en el que se configura la comunidad autónoma, se observa un aumento del nacionalismo andaluz.

Gráfico 1. Sentimiento andalucista y españolista de los andaluces (19822012)

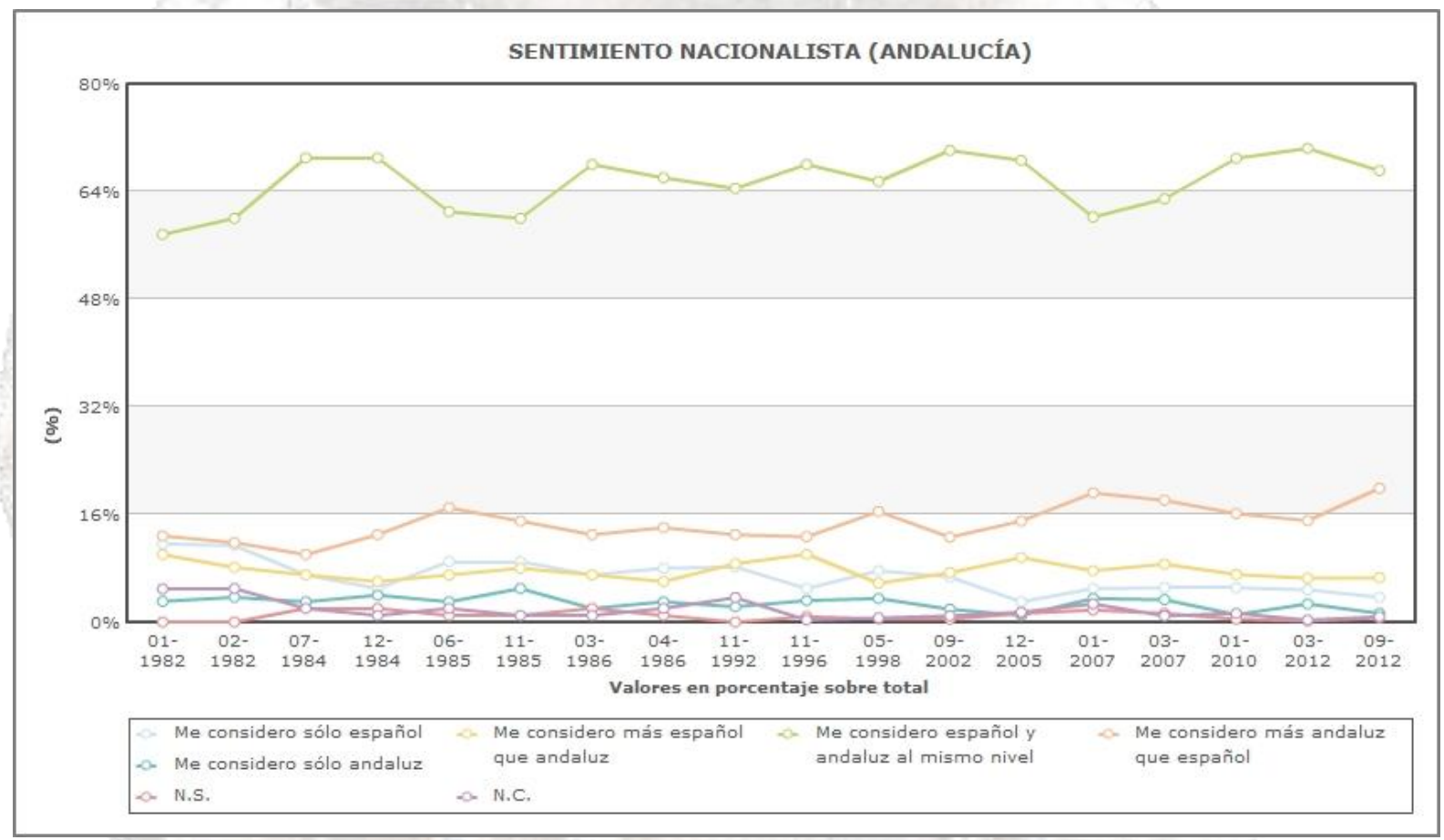

Fuente: Serie A.2.02.03.001, Banco de Datos, CIS.

En sintonía con estos datos, un reciente trabajo sostiene que el sentimiento de pertenencia a Andalucía es un hecho que tiene mayor relación con su expresión en la actual comunidad autónoma (Coller, 2014). Por un lado, distintas encuestas en las que se ha preguntado por esa identidad, demuestran como el nacionalismo andaluz 0 andalucismo actualmente es una posición minoritaria y de izquierdas (Aguiar y Espinosa, 2011: 22). Por lo que, la posición mayoritaria de la identidad andaluza corresponde con un sentimiento de naturaleza cultural desarrollado social e históricamente, pero que carece de un componente político. Según el Barómetro de Opinión Pública del IESA 
en 2005 , el $76,7 \%$ de los andaluces se sentía tan andaluz como español (Tabla I), sin que un sentimiento prevaleciese sobre el otro.

Tabla 1. Barómetro de Opinión Pública del IESA sobre sentimiento andaluz y español.

\begin{tabular}{|c|c|c|}
\hline Variable & Categorías & $\%$ \\
\hline \multirow{4}{*}{$\begin{array}{l}\text { Frase que expresa mejor } \\
\text { sus sentimientos }\end{array}$} & Sólo andaluz & 2,9 \\
\hline & Más andaluz que español & 10,3 \\
\hline & $\begin{array}{l}\text { Tan andaluz como } \\
\text { español }\end{array}$ & 76,7 \\
\hline & $\begin{array}{l}\text { Más español que } \\
\text { andaluz/Sólo español }\end{array}$ & 10,1 \\
\hline \multirow[t]{3}{*}{$\begin{array}{l}\text { Término para referirse a } \\
\text { Andalucía }\end{array}$} & $\begin{array}{l}\text { Es una comunidad } \\
\text { autónoma }\end{array}$ & 94,6 \\
\hline & Es una nación & 3,4 \\
\hline & Otro & 1,9 \\
\hline \multirow{3}{*}{$\begin{array}{l}\text { Preferencias para el } \\
\text { grado de autonomía de } \\
\text { Andalucía }\end{array}$} & Mayor & 46,3 \\
\hline & Igual & 51,4 \\
\hline & Menor & 2,3 \\
\hline \multirow{3}{*}{$\begin{array}{l}\text { Fórmulas alternativas de } \\
\text { organización del Estado } \\
\text { en España }\end{array}$} & $\begin{array}{l}\text { Estado con gobierno } \\
\text { central sin autonomías }\end{array}$ & 2,2 \\
\hline & $\begin{array}{l}\text { Estado con comunidades } \\
\text { autónomas como en la } \\
\text { actualidad }\end{array}$ & 86,9 \\
\hline & $\begin{array}{l}\text { Estado Federal con } \\
\text { CC.AA. } \\
\text { autonomías/Estado qués } \\
\text { reconozca la posibilidad } \\
\text { de convertirse en } \\
\text { independientes }\end{array}$ & 10,9 \\
\hline
\end{tabular}

Fuente: BOPA-2005. 
A la luz de estos datos, se observa como los andaluces se sentían satisfechos en su mayoría con la organización territorial de Andalucía dentro de España y el sentimiento que sentían tanto hacia su país como hacia su comunidad autónoma estaban jerárquicamente a la misma altura para fechas de la década pasada. Es interesante observar cómo entre las posiciones minoritarias en relación a las fórmulas alternativas de organización territorial, son superiores los federalistas que los centralistas. Por lo que, la práctica totalidad de los andaluces son opositores a retroceder en el grado de autonomía que ha adquirido Andalucía actualmente.

En términos similares, se muestra el análisis de la identidad andaluza realizado por Xavier Coller (2014: 50). Por su parte, sostiene que esta identidad carece de significación política y que tiene una dimensión territorialista. Por lo que, no es una condición necesaria el haber nacido en Andalucía, tener padres o familiares andaluces, sino que la identidad andaluza se construye como un sentimiento de pertenencia a un territorio determinado respecto a otros dentro del Estado de España.

Por tanto, se afirma que la identidad andaluza actualmente no ha adquirido un especial significado político y que se corresponde más con un sentimiento de pertenencia al territorio de la comunidad autónoma. Si bien, el llamado andalucismo o nacionalismo andaluz representa un sentimiento minoritario, apenas representado por una porción inferior al 5\% de la población (Aguiar y Espinosa, 2011; Del Pino y Bericat, 1996). Por lo que, la identidad andaluza coexiste pacíficamente con la identidad española para la mayor parte de los residentes en Andalucía.

En un trabajo de Bericat (2002: 71), se ponen en relación estos sentimientos con otras características culturales y sociales. Al respecto, señala que las posiciones más localistas y la minoría que sólo se siente andaluza se corresponde con una cultura tradicional y un estatus social bajo. Mientras que, los que destacan por un sentimiento más españolista o europeo, se corresponde con una cultura de corte posmoderno. Por lo que, concluye que una mayor identificación andaluza se corresponde con más tradicionalidad, y mayor identificación española se relaciona con una mayor posmodernidad.

\subsection{Identidades provinciales y locales: un recurso en manos de las élites municipales}

El debate sobre las identidades territoriales continúa con la consideración de identidades arraigadas a espacios físicos/niveles de gobierno de menor dimensión como la provincia y el municipio. Y es que, dentro de la misma comunidad autónoma existen otras identidades de carácter local o territorial que aluden a un espacio geográficamente inferior a Andalucía. En este sentido, la división entre la Alta Andalucía y la Baja Andalucía, o más nítidamente entre 
Andalucía Occidental y Andalucía Oriental ha producido la aparición de identidades respectivas en el siglo anterior y presente. En el caso de los andaluces orientales, esta identidad se ha construido como una oposición al centralismo político de Sevilla desde la creación de la comunidad autónoma. Por su parte, algunos políticos malagueños han recordado, en no pocas ocasiones, que el municipio de Antequera era la capital que originariamente se había previsto durante la junta preautonómica en los Pactos de Antequera. Un dato que desde el consistorio malagueño se suele recordar a la Junta de Andalucía de forma esporádica.

Aunque estas identidades locales y construidas en la rivalidad entre ciudades pueda parecer algo anecdótico o trivial, no debe ser subestimado. Si bien, no existen datos suficientes para conocer el peso de la identidad local o del sentimiento de pertenencia al municipio. Y esto es una laguna grave, porque especialmente los alcaldes han empleado discursos 12 basados en la defensa de la identidad local como oposición a otros municipios, especialmente Sevilla y la Junta de Andalucía por extensión, para denunciar y/o reclamar determinados recursos, infraestructuras o bienes. Por ejemplo, Francisco de la Torre (PP) cuando se opuso a las exigencias de ley de capitalidad de Sevilla ${ }^{13} \mathrm{o}$ cuando ha exigido una descentralización de las sedes de las instituciones autonómicas. A la inversa, los alcaldes de Sevilla y algunos consejeros de la Junta de Andalucía han promovido en varias ocasiones determinados discursos basados en su carácter como capital autonómica para exigir determinados servicios, sedes institucionales o recursos. Un caso paradigmático fue el caso de Ignacio Zoido (PP) cuando intentó plantear una ley de capitalidad ${ }^{14}$ ante el Parlamento Andaluz. O cuando se propuso, por parte del ejecutivo autonómico en 2009, la creación de una caja de ahorros única andaluza mediante la fusión de distintas entidades como Unicaja, CajaSol, CajaSur, Caja Granada, Caja Jaén y otras ${ }^{15}$. Lo que suscitó discrepancias y tiranteces entre los alcaldes y otros representantes locales de distintas provincias.

Por ello, las identidades locales y las oposiciones/rivalidades que se crean entre unas ciudades y otras y entre unas ciudades con respecto a

12 Como sostienen algunos autores (Díaz-Carrera, 2014; Jiménez-Díaz, 2008), los líderes tienden a crear una visión de la realidad y por tanto, uno de sus principales objetivos es crear un sentido de la realidad. Por lo que, las identidades locales son empleadas muchas veces cómo una forma de construir una perspectiva frente a otros territorios y/o municipios.

13 Para más información se puede consultar esta noticia publicada en El País: http://ccaa.elpais.com/ccaa/2012/08/03/andalucia/1344027789_319184.html

14 Otra información sobre un tema que ha continuado con la rivalidad entre Málaga y Sevilla se puede leer en este artículo de prensa: http://www.elconfidencial.com/espana/2011/09/18/guerra-civil-entre-sevilla-y-malagapor-la-ley-de-capitalidad-andaluza-84388.

15 Es posible obtener mayor información a partir de esta noticia: http://www.elmundo.es/elmundo/2009/07/13/andalucia/1247501510.html 
otros niveles de gobierno son aprovechados como instrumentos de los líderes locales para hacer prevalecer los intereses del municipio, para fortalecer sus redes de seguidores y aumentar su apoyo electoral en el mismo. Por lo tanto, un hecho indudable de estas identidades es que son socialmente y políticamente construidas a lo largo de períodos históricos. Si bien, detrás de este discurso polémico sobre la rivalidad entre las ciudades andaluzas, se esconde lo que algunos han venido en llamar como el "desarrollo asimétrico", "desarrollo desigual" o los "desequilibrios internos" de Andalucía (Entrena, 2006; Domínguez Ortíz, 1983; Lacomba, 1995; Navarro y Pérez-Yruela, 2001).

\subsection{La identidad europea entre los andaluces}

Finalmente, el sentimiento de pertenencia a la Unión Europea representa otra identidad territorial que tampoco debe olvidarse en este análisis de la territorial desde la subjetividad de los andalúces. Si bien, al no disponer de datos o barómetros que lo midan a nivel regional, se puede observar la serie del CIS sobre identidad europea por parte del conjunto de los españoles (Gráfico 2).

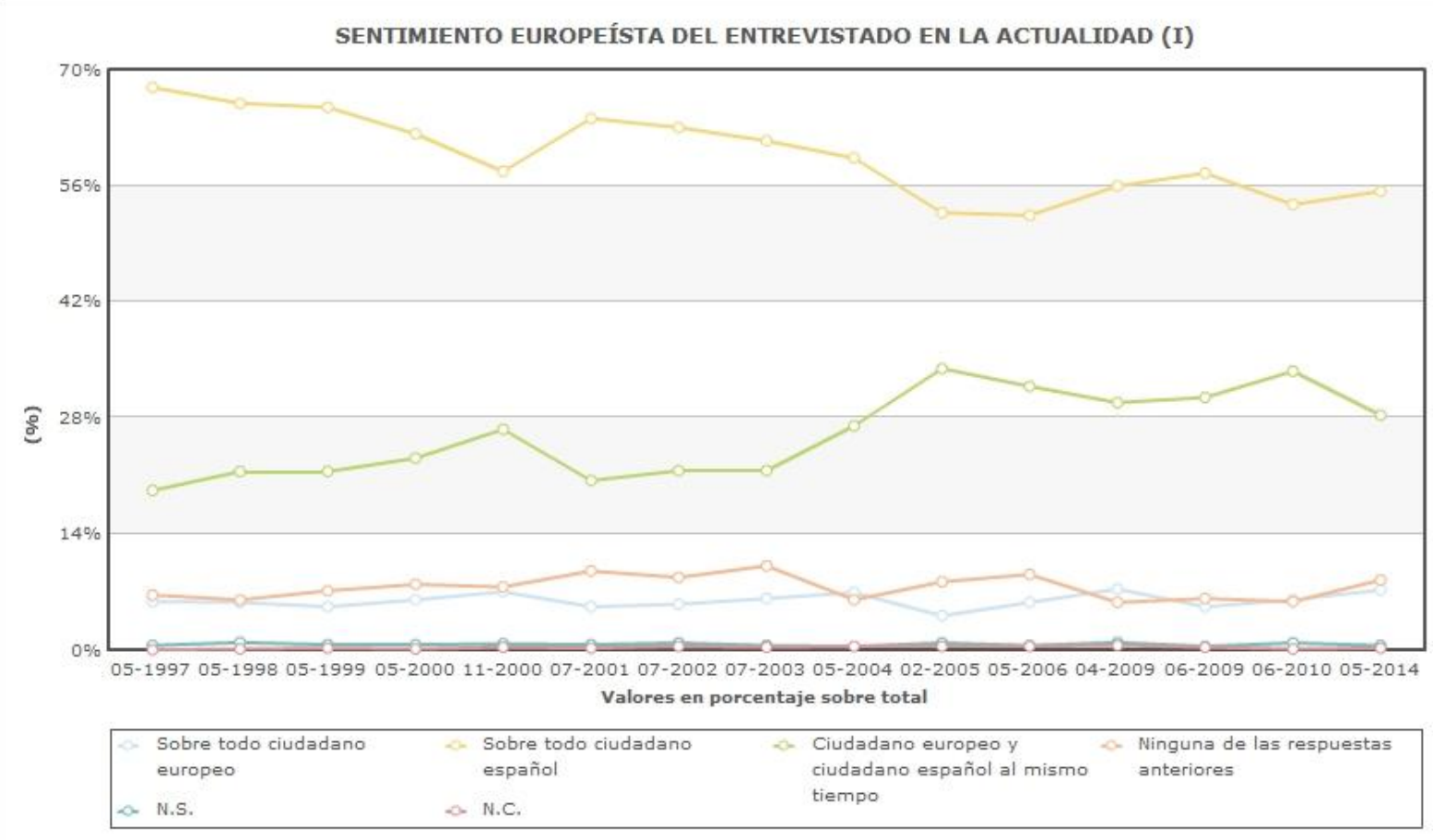

Gráfico 2. Sentimiento europeísta de los españoles (1997-2014)

Fuente: Serie C.2.04.01.001, Banco de Datos, CIS.

A la luz de estos datos se observa como el sentimiento de pertenencia al Estado español por encima de la ciudadanía europea es la opinión 
mayoritaria a lo largo del período, no situándose en ningún momento por debajo del $50 \%$. Por lo que, se mantiene la importancia del Estadonación como institución con la que se identifican los españoles, pese a que haya perdido potestades en el ámbito económico y monetario. La segunda opinión imperante es la de aquellos que se sienten tanto ciudadanos europeos como ciudadanos españoles.

Además, el sentimiento europeísta es reducido y escaso entre los sujetos, no llegando a lograr el $5 \%$ aquellos que se sienten ante todos miembros de la Unión Europea. Probablemente, el fracaso de la Constitución Europea y la insolidaridad territorial entre los países miembros durante la crisis económica de 2008 hayan influido determinantemente a que no hayan cambiado las tendencias en lo que respecta al sentimiento de pertenencia a la organización comunitaria.

Según álgunos análisis, el apoyo a la Unión Europea está relacionado con altos niveles de formación, determinadas profesiones y prácticas que exigen viajar y el contacto o la amistad con personas de otros países comunitarios (García Faroldi, 2004). Asimismo, subraya que no existe una relación entre nivel económico y sentimiento europeísta, pues existen casos de ciudadanos con una renta alta, pero que están inmersos en una red social donde son mayoritarios los sentimientos más regionalistas $y / 0$ localistas y esto determina una menor identidad europea.

\section{Valores políticos y cultura política en Andalucía}

En este apartado de lo que hemos denominado como la dimensión simbólico-cultural se realiza un análisis de los valores y actitudes políticas imperantes entre la sociedad andaluza en la evolución cultural desde la tradicionalidad hacia la "postmodernidad" o "modernidad"16, que puede corresponderse con la actual demoracia. La literatura científica sobre los valores es bastante amplia, pues representa un ámbito de estudio interdisciplinar donde tienen cabida las ópticas de la Cíencia Política, la Sociología, la Antropología, la Psicología y la Filosofía entre otros saberes. Es por ello, que es necesario delimitar cuáles son los valores de los que se va a hablar en este apartado.

Cuando la Ciencia Política ha abordado los valores políticos se ha centrado principalmente en las actitudes políticas o la llamada cultura política. ¿Qué es la cultura política? Según Parsons y a partir de Weber, la cultura política comprende un conjunto de actitudes, comportamientos y sentimientos subjetivos que caracterizan las orientaciones de los sujetos hacia un sistema político (Somers, 1996: 45-

16 Algunos autores prefieren hablar de "modernidad" (Entrena, 2001), mientras que otros acuñan el reciente concepto de "postmodernidad" (Giddens y otros, 2008) para referirse a la época que vive la actual sociedad según la asunción de una serie de valores postmaterialistas. 
52). Por su parte, Almond y Verba en su clásico The Civic Culture afirman que la cultura política está compuesta por tres tipos de orientaciones: cognitivas (conocimientos del sistema político y sus inputs-outputs), afectivos (sentimientos hacia el sistema político y sus actores) y evaluativas (juicios y opiniones sobre el sistema político y sus actores). Así, la cultura política sería "una particular distribución de los patrones de orientaciones hacia objetos políticos entre los miembros de una nación" (Almond y Verba, 1965: 15). Más tarde, han aparecido nuevas aportaciones como la "nueva cultura política" o "cultura postmoderna", en los que aspectos como el tradicional eje izquierdaderecha pierden peso y los valores materialistas dan paso a los postmaterialistas como resultado de cambios en la situación económica, la familia y el creciente peso de los medios de comunicación (Inglehart, 1990; Merelman, 1998; Nichols Clark y Hoffman-Martinot, 1998; Putnam, 1993).

Por parte de otras disciplinas como la Psicología, el clásico psicólogo social Festinger en su teoría de la disonancia cognitiva afirma que las personas buscan un equilibrio subjetivo entre sus valores y expectativas y la situación de su realidad externa. Si en algún momento, se produjese una diferencia radical entre esos valores y sus actitudes, el sujeto busca acabar con dicha disonancia mediante el cambio de sus valores o de sus acciones (Festinger, 1975). También, existe una reciente corriente que reafirma el valor de los sujetos y sostiene que el valor hace que el individuo soporte determinadas situaciones que están al servicio de esos valores (Hayes, Barnes Holmes y Roche, 2001).

Desde posturas más interdisciplinares, los contructivistas afirman que los valores son una construcción social creada como resultado de la vida en colectivo (Parsons 2010). No habría una diferencia en cuanto a su proceso de creación subjetiva entre un valor y una institución. Así, los valores además de ser algo artificial se enmarcan en conjuntos organizados de valores y criterios de juicio creando un marco (Goffman 2009). Los marcos son subuniversos de la subjetividad de cada persona que permiten ordenar su percepción de la realidad, diferenciar lo importante de lo superfluo, construir un discurso sobre la realidad externa y además distinguir lo correcto de lo incorrecto según dicho discurso (Collado-Campaña, Jiménez Díaz y Molero, 2010; MartínCriado, 1998).

Ante tan diversas aportaciones, se procede a la realización de una definición de valores para nuestra investigación. Se define un valor será aquel elemento de la subjetividad interor de la persona que permite distinguir lo correcto y lo incorrecto. Si bien, su concreción en una actitud hacia un elemento concreto de la realidad efectiva es lo que denominados como opinión, siendo una actitud en la que influye de forma determinante el contexto. Una vez, se ha zanjado esta cuestión, se procede a la selección de una serie de valores y actitudes de especial interés para analizar en el contexto de Andalucía en el período 
estudiado, a saber: la valoración de la situación de la comunidad autónoma, el eje izquierda-derecha de autoubicación ideológica y los valores materialistas y postmaterialistas de sus habitantes.

\section{1. ¿Cómo va la cosa por Andalucía? Valoración de la realidad politica de la autonomía}

En las series del CIS de valoración de la situación política de Andalucía (Gráfico 3) se puede observar como la valoración mayoritaria de los andaluces era una opinión "regular" cuando se les preguntaba por la situación política de las instituciones autonómicas . Si bien, esta opinión que se ha mantenido entre un 36\% y un 55\% ha disminuido desde 2004 hasta la actualidad para pasar a ser la segunda postura mayoritaria, y siendo la opinión de "mala" la imperante para finales de 2010 y comienzos de 2011 . Por su parte, la opinión positiva se ha mantenido por debajo del $25 \%$ durante la década de los noventa y la mayor parte del período, salvo en dos momentos puntuales en 2004 y 2008 que ha repuntado, probablemente coincidiendo con la extensión de competencias del Gobierno autonómico y la redacción del nuevo estatuto de autonomía. Para finales de nuestro plazo temporal, se observa como la situación "mala" o "muy mala" son compartidas por más del $60 \%$ de los andaluces. A grandes rasgos, no ha existido una valoración positiva de los andaluces de la evolución política de la autonomía.

Gráfico 3. Valoración de la situación política actual de la comunidad autónoma de Andalucía (1994-2012).

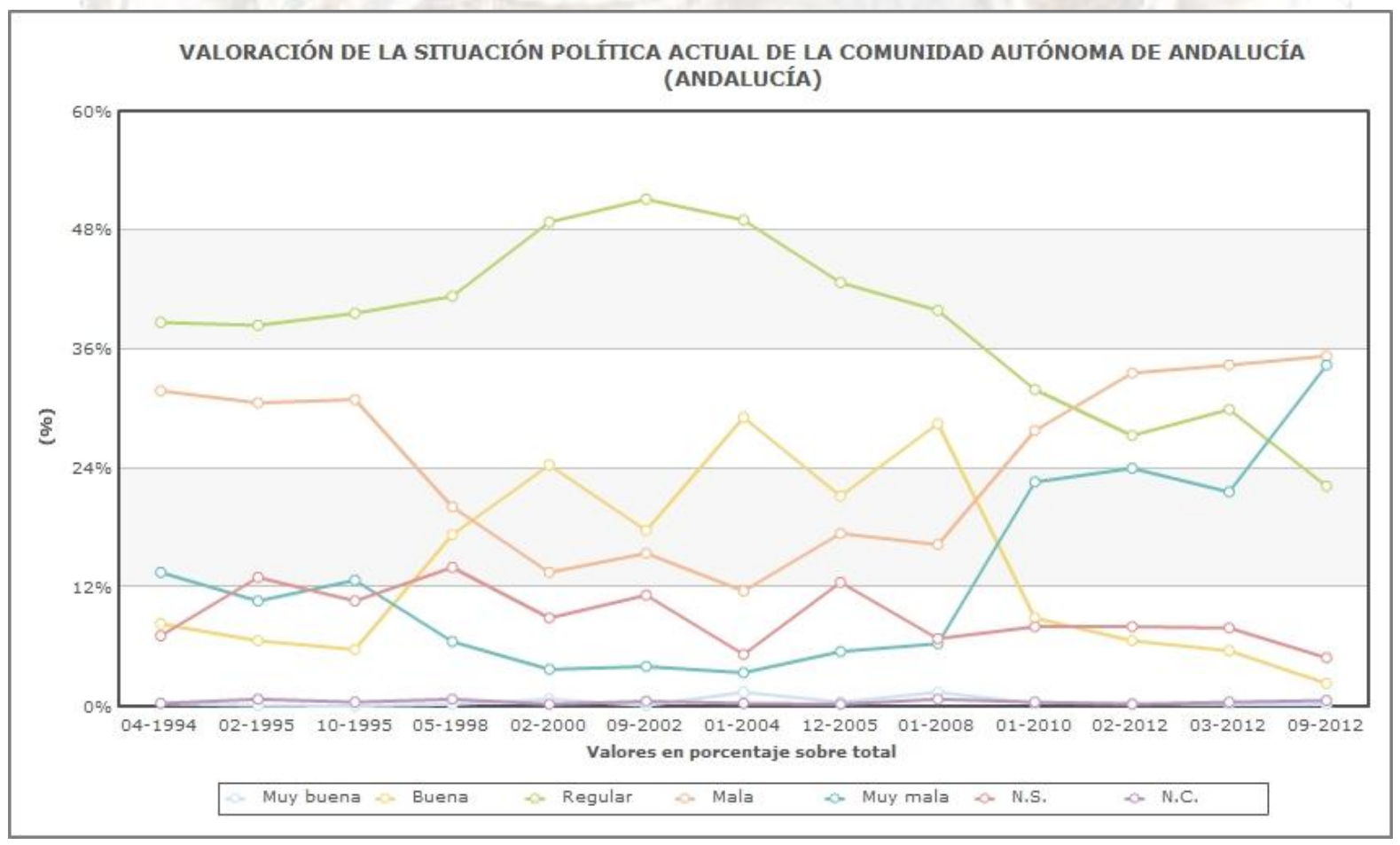




\section{2. ¿De izquierdas o de derechas?}

¿Son los andaluces de izquierdas o de derechas? De entrada, la mayoría de los andaluces se consideran de centro-izquierda (3-4) cuando se ubican en el eje ideológico, situándose entre un 30-40\% a excepción del período comprendido entre 1996 y 2005 (Gráfico 4). Durante dicha época, los andaluces que se declararon de centro (5-6) llegaron a superar en momentos concretos a los de centro-izquierda. Así pues, el centro ha sido la segunda posición mayoritaria y se puede observar como ha experimentado un crecimiento a lo largo de los noventa y posteriormente a partir de 2007. Por otro lado, la posición de centro-derecha (7-8) es bastante minoritaria representando siempre una cifra entre $11-5 \%$. Las posiciones más extremas se mantienen por lo general por debajo del $10 \%$, aunque los andaluces de extrema izquierda (1-29 superan a los de extrema derecha (9-10) en la totalidad de la serie.

A la luz de estos datos, la mayoría de los andaluces son ciudadanos de centro-izquierda y centro en el eje de auto-ublicación ideológica. Esto puede explicar en parte la continuidad ininterrumpida del PSOE en el Gobierno de la Comunidad Autónoma, ya que como partido socialdemócrata su principal caladero de votos ha permanecido en el centro-izquierda y el centro, obteniendo puntualmente votos del extremo del extremo izquierda y produciendo cierta volatilidad períodica entre el electorado tradicional de IU. De esta forma, el perfil del votante promedio en Andalucía es similar al del conjunto del Estado español.

\section{Gráfico 4. Escala de autoubicación ideológica (1-10) de los andaluces (1984-2012).}

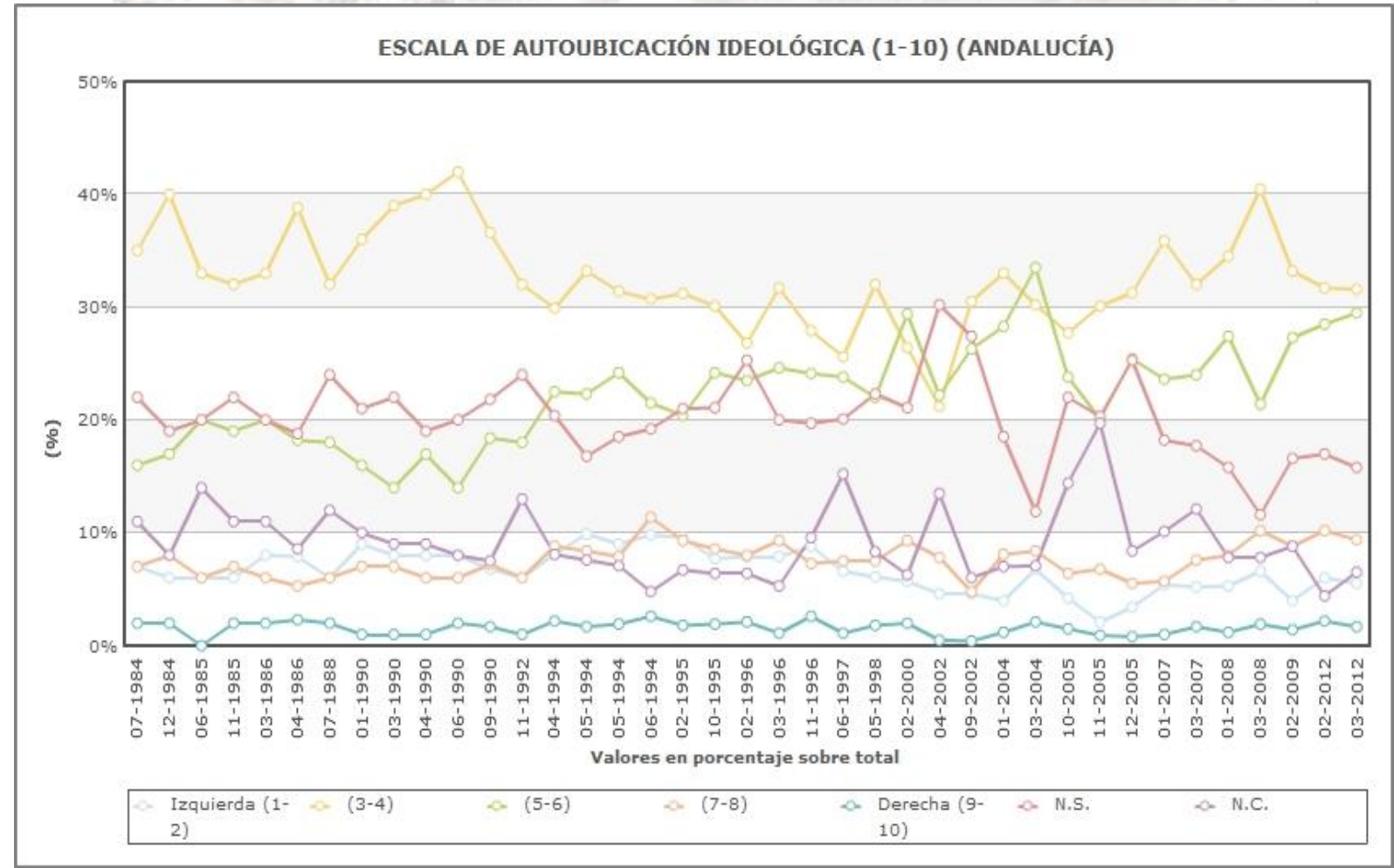




\subsection{El postmaterialismo que no termina de cuajar}

El postmaterialismo del que hemos hablado anteriormente, se corresponde con la teoría de la modernización desarrollada especialmente por algunos estudiosos (Inglehart, 1990; Putnam, 1993). A partir de sus estudios empíricos, concluyen que a finales del siglo XX se ha producido un cambio cultural y la aparición de unos nuevos valores como la protección de los derechos civiles, la búsqueda de una sociedad menos impersonal, la integración de las personas en la comunidad, la auto-realización, la preocupación por el medio ambiente y la solidaridad altermundista.

Hipotéticamente, estos principios irán sustituyendo paulatinamente a los valores materialistas como la seguridad personal, la propiedad y la cobertura de las necesidades más básicas para el sustento humano. Esto se produciría a partir de un reemplazo generacional después de la Il Guerra Mundial, en aquellos países con mayor renta pér cápita. Por lo que, esta teoría sostiene que conforme se produce un determinado aumento del PIB de una sociedad, son mayores las personas con valores postmaterialistas que aquellos en los que priman los materialistas. Este cambio influye en múltiples aspectos políticos como los factores que determinan el voto y sociales como la participación cívica y/o el consumo cultural.

Ahora bien, es una teoría que todavía no ha terminado de consolidarse a partir de la reciente crisis económica como resultado de la inseguridad social (Díez Nicolas, 2007) o bien porque los procesos de modernización no son homogéneos ni simultáneos en el tiempo debido a las circunstancias de cada territorio (Entrena, 2001). Y es que, el postmaterialismo aparece a partir del momento en que una sociedad consigue resolver las necesidades materiales de la mayoría de sus habitantes. En este sentido, surge la pregunta de cuál es la situación de esto valores entre los andaluces.

Ante esta discusión sobre los valores postmaterialistas y materialistas no hay datos suficientes para conocer en profundidad su implantación entre los andaluces. Si bien, existen distintas aproximaciones por parte de algunos investigadores que permiten dibujar una aproximación sobre dichos interrogantes a partir de la década de los noventa, ya que es difícil hablar de modernización en España antes de dicha época. En un trabajo comparativo del postmaterialismo entre los andaluces y catalanes (Fernández Alonso, 2010), se observa como en ninguna de las dos comunidades autónomas los postmaterialistas superan al resto (Tabla II). No obstante, el grupo de postmaterialistas es mayor en Cataluña que en Andalucía para los dos años observados. Lo realmente destacable es como en Andalucía se ha reducido la minoría postmaterialista en el paso de los noventa a la primera década del siglo XXI, cuando además se había producido un incremento del PIB durante 
esos años. Lo que refuta la hipótesis de la modernidad en la comunidad autónoma.

Tabla 2. Población materialista y postmaterialista en porcentajes por filas, según comunidad autónoma y año.

\begin{tabular}{|c|c|c|c|c|}
\hline & Materialistas & Mixtos & $\begin{array}{l}\text { Postmaterialist } \\
\text { as }\end{array}$ & Total (N) \\
\hline \multicolumn{5}{|l|}{1999} \\
\hline Andalucía & 23,4 & 67,4 & 9,2 & 100 (892) \\
\hline Cataluñ & 15,1 & 71,9 & 13 & $\begin{array}{l}100 \\
(1.064)\end{array}$ \\
\hline \multicolumn{5}{|l|}{2004} \\
\hline Andalucía & 25,8 & 70,7 & 3,5 & $100(518)$ \\
\hline Cataluña & 11,7 & 73,2 & 15,1 & $100(639)$ \\
\hline
\end{tabular}

Fuente: Fernández Alonso, 2010: 1038.

Por lo tanto, los valores postmaterialistas no se han impuesto entre la sociedad andaluza. Al contrario, es más adecuado hablar de una convivencia entre valores materialistas y posmaterialistas, que es la postura compartida por más del $60 \%$ de los andaluces a la luz de estos datos. Ahora bien, se puede afirmar que existe un aumento de los valores postmaterialistas frente a lo materialistas en los siguientes casos: el hábitat urbano frente al rural, las clases superiores frente a las inferiores, los niveles de formación más altos con respecto a los más bajos, entre las personas que se encuentran en el mercado laboral frente a las que están fuera y entre las personas de menor edad frente a los más mayores (Fernández Alonso, 2010: 1039-1045). En esta última variable, lo determinante no es tanto la edad, sino el reemplazo de una generación más formada y con procesos y agentes de socialización distintos a los más mayores.

\section{A modo de conclusión: una ciudadanía moderada y políticamente fatalista}

En síntesis, se puede obtener una visión global de las identidades territoriales y de la cultura política a partir de los datos expuestos. En el primero de los elementos analizados, se observa como casi dos tercios de los andaluces se sienten tanto españoles como parte de su comunidad autónoma a lo largo de estas cuatro décadas de 
democracia. El nacionalismo andaluz o la existencia de un sentimiento regionalista más intenso es un elemento prácticamente minoritario. Al respecto hay que tener en cuenta dos factores para entender el escaso arraigo del sentimiento andalucista. Por un lado, el fracaso del andalucismo como opción política partidista y el imaginario construido de Andalucía a través del turismo y los medios de comunicación. Por otro lado, el éxito catalán al crear una identidad nacionalista a través de la literatura, la cultura y la existencia de partidos políticos estables con dicho carácter. En este sentido, se puede sostener ve es un problema de "construcción" en tanto que las identidades territoriales son elementos creados socialmente y no tienen un carácter natural. De ahí, que la identidad andaluza mayoritaria esté más relacionada con la pertenencia a un ente subterritorial, la comunidad autónoma, y no con el hecho de haber naciado, compartir una historia común o la existencia de una determinada tradición.

Mientras que, las posiciones minoritarias como sentirse más español que andaluz, más andaluz que español, sólo andaluz o sólo español son prácticamente inexistentes. Ahora bien, es arriesgado afirmar que una posición equilibrada de ambas identidades esté relacionada con posiciones postmaterialistas o con entornos urbanos y viceversa, ya que esto no deja de ser una propuesta normativa para legitimar unas características socio-demográficas como mejores que otras. De hecho, estas afirmaciones deberían evitar dicho riesgo generalizador a través de distintos estudios de casos y trabajos cualitativos que pudiesen refrendar o rechazar dichos enunciados generados a través de lo cuantitativo.

En relación a las identidades locales no se han encontrado datos desglosados a nivel andaluz ni mucho menos provincial-local, pero se ha observado como el empleo de determinados discursos identitarios por parte de la clase política local ha sido empleado (y son empleados) en determinados conflictos entre municipios y/o entre nivel de gobierno local contra el autonómico. Por lo que, serían deseables bases de datos o estudios que recabaran de forma desglosada estos datos hasta el nivel provincial especialmente para los politólogos, sociólogos y antropólogos. Finalmente, algo similar sucede con el sentimiento de pertenencia a la Unión Europea, donde se echa en falta la existencia de series temporales desglosadas para el nivel andaluz. En este aspecto, la preeminencia de la identidad nacional es claramente mayoritaria a lo largo de todo el período. Pese a los efectos beneficiosos que Europa ha tenido para España y Andalucía, sólo se observa un ligero aumento del sentimiento europeísta y del sentimiento nacionalista para el conjunto español, respectivamente.

En el ámbito de la cultura política, la mayoría de los andaluces han tenido mayoritariamente una opinión regular y mala de la realidad política de Andalucía, jerarquía que se ha invertido en los últimos años con el empeoramiento de la crisis económica. Políticamente, el 
ciudadano andaluz promedio se sitúa en el centro-izquierda y el centro como muestra de una ideología política moderada. Es interesante observar cómo los andaluces que se declaran de centro político han aumentado en los últimos años y han disminuido ligeramente los que se sienten de centro-izquierda. Por lo que, durante la crisis económica el peso de estas cuotas ideológicas han avanzado hacia una posición que podríamos calificar de "equilibrada" o "centrista". Un factor que sería interesante analizar a partir de otros estudios y especialmente, análisis cualitativos de dichos grupos ideológicos. Finalmente, la existencia del llamado "postmaterialismo" es prácticamente inexistente y la mayoría se define por tener unos valores híbridos entre la seguridad y la libertad. Lo que lleva a poner en tela de juicio las teorías de la modernidad que muchas veces son enunciadas desde planteamientos normativistas.

En síntesis, el perfil mayoritario andaluz para estas cuatro décadas de democracia se corresponde con una persona: que se siente tanto española como andaluza, en menor grado europea, que percibe una situación política regular en su comunidad autónoma, que ideológicamente es de centro-izquierda y que mantiene una postura intermedia entre los elementos materialistas y postmaterialistas. De esta forma, uno de los mejores calificativos para este ciudadano es el de un carácter políticamente moderado y con un sentimiento de pertenencia más cívico que étnico, mientras convive con un panorama político que no termina de evaluar como positivo.

\section{Referencias bibliográficas}

Aguiar, F. y Espinosa, E. (2011). Identidad andaluza y realidad nacional. Córdoba: IESA.

Almond, G. y Verba, S. (1965). The civic culture: political attitudes and democracy in five nations. An analytic study. Boston: Little, Brown and Company.

Bericat, E. (2002). Valores tradicionales, modernos y posmodernos en la sociedad andaluza. En E. Moyano \& M. Pérez-Yruela (eds.), La sociedad andaluza [2000] (pp. 45-64). Córdoba: IESA.

CIS. Banco de Datos. Madrid: CIS. Consultado de http://www.analisis.cis.es/cisdb.jsp.

Collado-Campaña, F. (2012). Bizancio y la provincia de Spania: ¿̇capital Málaga?. Gibralfaro: Revista de Humanidades y Creación Literaria, 75. 
Collado-Campaña, F.; Jiménez-Díaz, J.F. y Molero, J.A. (2010). El estudio del discurso político: una aproximación desde la Sociología y la Lingüística. En R. Cremades, C. González y S. Guerrero (eds.), Estudios actuales sobre Lengua, Literatura y su Didáctica: en Homenaje a Emilio Núñez Cabezas (pp. 23-45). Málaga: Universidad de Málaga.

Coller, X. (2014). Factoría de ideas: Perspectivas sobre la identidad andaluza. Sevilla: Centro de Estudios Andaluces (Junta de Andalucía).

Cuenca, J.M. (1984). La Andalucía de la transición (1975-1984): política y cultura. Madrid: Mezquita.

De los Santos, José María (2002). Andalucía en la transición (1976-1982). Sevilla: Centro de Estudios Andaluces.

Del Pino, J. y Bericat, E. (1996). Valores sociales en la cultura andaluza. Madrid: CIS.

Díaz-Carrera, C. (2014). El líder como generador de sentido. En C. DíazCarrera y A. Natera (eds.), El coraje de liderar (pp. 187-219). Madrid, Tecnos.

Díez Nicolás, J. (2007). ¿Regreso a los valores materialistas?: El dilema entre seguridad y libertad en los países desarrollados. Actas del IX Congreso Español de Sociología "Poder, cultura y civilización Barcelona: FES.

Domínguez Ortiz, A. (1983). Andalucía ayer y hoy. Barcelona: Planeta/ Instituto de Estudios Económicos.

Entrena F. (2006). Diversificación de las desigualdes y estabilización social en Andalucía. Granada: Universidad de Granada.

Entrena, F. (2001). Modernidad y cambio social. Madrid: Trotta.

Fernández Alonso, M. (2010). Valores y creencias en el proceso de la modernización. Papers, 95, 1031-1051.

Festinger, L. (1975). Teoría de la disonancia cognitiva. Madrid: Centros de Estudios Políticos y Constitucionales.

García Faroldi, L. (2009). Nación y territorio. Análisis comparado de sentimientos nacionalista y la pertenencia a territorios en Andalucía y Cataluña. Papers, 95(4), 911-934.

García Faroldi, L. (2004). Apoyo a la Unión Europea y difusión de una identidad europea a través de las redes personales. Redes: Revista hispana para el análisis de redes sociales, 7.

Goffman, E. (2009). La representación de la persona en la vida cotidiana. Buenos Aires: Amorrortu.

Hayes, S.C., Barnes-Holmes, D. and Roche, B. (2001). Relational Frame Theory: A Post-Skinnerian account of human language and cognition. New York: Plenum Press. 
Hijano del Río, M. y Ruiz Romero, M. (1997). El pacto autonómico de Antequera: (4 de diciembre de 1978): un documento para la historia de Andalucía. Sevilla: Instituto Andaluz de Administración Pública (Junta de Andalucía).

Inglehart, R. (1990). Culture shift in advanced industrial society. Princeton: Princeton University Press.

Jerez, M. (1985). Una experiencia de partido regional: el caso del Partido Socialista de Andalucía. REIS: Revista española de investigaciones sociológicas, 30, 201-244.

Lacomba, J.A. (1995). La economía andaluza: un enfoque desde la historia. Sevilla: Instituto de Desarrollo Regional.

Linz, J.J. (1985). De la crisis de un Estado unitario al Estado de las autonomías. En F. Fernández (ed.), La España de las Autonomías, editado por F. Fernández. Madrid: Instituto de Estudios de Administración Territorial.

Moreno Navarro, I. (1998). La identidad histórica y cultural de Andalucía. Biblioteca Andaluza (Nación Andaluza). Descargado de http://www.nacionandaluza.info/biblioteca\%20andaluza/IsidoroMorenoLaidentidadhistoricayculturaldeAndalucia.pdf

Moreno Navarro, I. (1994). Andalucía: identidad y cultura (Estudios de Antropología Andaluza). Málaga: Ágora.

Moyano, E. y Pérez-Yruela, M. (2002). La sociedad andaluza [2000]. Córdoba: IESA.

Navarro, C. y Pérez-Yruela, M. (2002). Cultura política en Andalucía. En E. Moyano \& M. Pérez-Yruela (eds.), La sociedad andaluza [2000] (pp. 109123). Córdoba: IESA.

Nichols Clark, T. y Hoffman-Martinot, V. (1998). The new political culture. Boulder: Westview Press.

Parsons, C. (2010). Constructivism and interpretative theory. En D. Marsh and G. Stoker (eds.), Theory and Methods in Political Science (pp. 80-98). London: Macmillan.

Putnam, R. (1993). Making democracy work: civic traditions in modern Italy. Princeton: Princeton University Press.

Rojas Marcos, A. (2004). Contra el letargo andaluz: Andalucía ante la revolución global, la nueva Europa y la España asimétrica. Córdoba: Almuzara.

Rovira, A. (1990). La renaixença catalana. Barcelona: Abadía de Montserrat.

Ruiz Muñoz, M.J. y Sánchez Alarcón, I. (2008). La imagen de la mujer andaluza a través del cine español. Sevilla: Centro de Estudios Andaluces. 
Ruiz Romero, M. (2002). La constitución de la Junta de Andalucía en la génesis del Estado de las autonomías. Hispania Nova: Revista de historia contemporánea, 2.

Somers, M. R. (1996). ¿¿Qué hay de político o de cultural en la cultura política y en la esfera pública? Hacia una sociología histórica de la formación de conceptos. Zona abierta, 77-78, 31-94.

Sánchez Alarcón, I.; Díaz, M.; Martín, F. y Ruiz, M.J. (2007). Los personajes como elementos configuradores del género musical español en sus inicios (1934-1936). Razón y palabra, 56.

Vega, M. (2010). Joan Triadú, repás d'una vida fecunda. Serra d'Or , 612, 33-40. 